\title{
LOWER BOUND FOR THE NUMBER OF REAL ROOTS OF A RANDOM ALGEBRAIC POLYNOMIAL
}

\author{
M. N. MISHRA, N. N. NAYAK and S. PATTANAYAK
}

(Received 21 January 1982; revised 24 May 1982)

Communicated by R. L. Tweedie

\begin{abstract}
Let $X_{1}, X_{2}, \ldots, X_{n}$ be identically distributed independent random variables belonging to the domain of attraction of the normal law, have zero means and $\operatorname{Pr}\left\{X_{r} \neq 0\right\}>0$. Suppose $a_{0}, a_{1}, \ldots, a_{n}$ are non-zero real numbers and $\max _{0<r<n}\left|a_{r}\right|=k_{n}, \min _{0<r<n}\left|a_{r}\right|=t_{n}$ and $\varepsilon_{n}$ is such that as $n \rightarrow \infty$, $\varepsilon_{n} \rightarrow 0$, but $\varepsilon_{n} \log n \rightarrow \infty$. If $N_{n}$ be the number of real roots of the equation $\sum_{r=0}^{n} a_{r} X_{r} x^{r}=0$ then for $n>n_{0}, N_{n}>\varepsilon_{n} \log n$ outside an exceptional set of measure at most $\mu / \varepsilon_{n} \log n+$ $\left(k_{n} / t_{n}\right)^{\beta} \exp \left(-\mu^{\prime} \beta / \varepsilon_{n}\right),(0<\beta<2-\varepsilon, 0<\varepsilon<2)$ provided $\lim _{n \rightarrow \infty}\left(k_{n} / t_{n}\right)$ is finite.
\end{abstract}

1980 Mathematics subject classification (Amer. Math. Soc.): 05 A 17, 05 A 19.

\section{Introduction}

Let $N_{n}(u)$ be the number of real roots of a random algebraic equation $\sum_{r=0}^{n} X_{r}(u) x^{r}=0$ where $X_{r}$ 's are independent identically distributed random variables. The problem of finding bounds for $N_{n}(u)$ has been considered by various authors. Samal (1972) has considered the general case when $X_{r}^{\prime}$ 's have identical distribution with expectation zero, variance and third absolute moment finite and non-zero. A stronger result has been obtained by Littlewood and Offord (1939) in the case where the coefficients are Gaussian variates. Dunnage $(1968,1970,1972)$ while dealing with general probability distribution has considered the lower bound of $N_{n}(u)$. Almost all the previous workers have considered the cases in which the random variables have finite moments of second and higher orders. The exception to those are the studies of Samal and Mishra (1972a, 1972b, 1973 ) in which they have considered the identically distributed random variables having characteristic function $\exp \left(-C|t|^{\alpha}\right)$ where $C$ is a positive constant and

(9) Copyright Australian Mathematical Society 1983 
$\alpha>1$. The probability distribution in this case represents a symmetric stable distribution with infinite variance when $1<\alpha \leqslant 2$.

The lower bound for the number of real roots of a random algebraic equation when $X_{r}$ 's belong to the domain of attraction of the normal law has not yet been studied, though Ibragimov and Maslova (1971) have studied the expectation of the number of real roots for this situation. The object of this paper is to find the lower bound of $N_{n}(u)$ when the coefficients are not identically distributed and belong to the domain of attraction of the normal law.

Theorem 1. Let $f(x)=\sum_{r=0}^{n} a_{r} X_{r} x^{r}$ be a polynomial of degree $n$ where the $X_{r}^{\prime} s$ are identically distributed independent random variables which belong to the domain of attraction of the normal law, have zero means and $\operatorname{Pr}\left\{X_{r} \neq 0\right\}>0$. Let $a_{0}, a_{1}$, $a_{2}, \ldots, a_{n}$ be non-zero real numbers. Then there exists a positive integer $n_{0}$ such that for $n>n_{0}$, the number of real roots of the equations $f(x)=0$ is at least $\varepsilon_{n} \log n$ outside a set of measure at most

$$
\mu / \varepsilon_{n} \log n+\left(k_{n} / t_{n}\right)^{\beta} \exp \left(-\mu^{\prime} \beta / \varepsilon_{n}\right), \quad(0<\beta<2-\varepsilon, 0<\varepsilon<2),
$$

provided $\lim _{n \rightarrow \infty}\left(k_{n} / t_{n}\right)$ is finite, where $k_{n}=\max _{0 \leqslant r \leqslant n}\left|a_{r}\right|, t_{n}=\min _{0 \leqslant r \leqslant n}\left|a_{r}\right|$ and $\varepsilon_{n} \rightarrow 0$, but $\varepsilon_{n} \log n \rightarrow \infty$ as $n \rightarrow \infty$.

In the sequel we need the following definitions, notation and lemmas for the proof of the theorem.

We shall denote $\mu$ 's for positive constants not necessarily the same in different places of occurrence. $[x]$ denotes the greatest integer $\leqslant x$. We take constants $A$ and $D$ satisfying the relations

$$
0<D<1 \text {, and } A>1 \text {. }
$$

Following the techniques of Samal and Mishra (1972a) we consider $f(x)=$ $\sum_{r=0}^{n} a_{r} X_{r} x^{r}$ at the points

$$
x_{m}=\left(1-M^{-2 m}\right)^{1 / 2},
$$

for $m=[k / 2]+1,[k / 2]+2, \ldots, k$, where

$$
M=\left[\lambda_{n}^{2}(\sqrt{2}+1)^{2}(A e / D)\left(k_{n} / t_{n}\right)^{2}\right]+1,
$$

$k$ is determined by

$$
M^{2 k} \leqslant n<M^{2 k+2},
$$

and $\lambda_{n}$ is a sequence tending to infinity with $n$. 
We express $f\left(x_{m}\right)$ for sufficiently large $n$ as sum of three parts as follows:

$$
f\left(x_{m}\right)=\left(\sum_{1}+\sum_{2}+\sum_{3}\right) a_{r} X_{r}(u) x_{m}^{r},
$$

where the index $r$ ranges from $M^{2 m-1}+1$ to $M^{2 m+1}$ in $\Sigma_{1}$, from 0 to $M^{2 m-1}$ in $\Sigma_{2}$ and from $M^{2 m+1}+1$ to $n$ in $\Sigma_{3}$.

We write

$$
U_{m}=\sum_{1} a_{r} X_{r}(u) x_{m}^{r}
$$

and

$$
R_{m}=\left(\sum_{2}+\sum_{3}\right) a_{r} X_{r}(u) x_{m}^{r} .
$$

We say a function $h: \mathbf{R}_{+} \rightarrow \mathbf{R}_{+}$is slowly varying in the neighbourhood of zero if

$$
\lim _{x \rightarrow 0} h(\nu x) / h(x)=1, \quad(\nu>0) .
$$

LEMMA 1. A slowly varying function $h$ with property (2.7) can be represented in the form

$$
h(x)=c(x) \exp \left\{-\int_{a}^{x} \frac{\bar{\varepsilon}(u)}{u} d u\right\}
$$

where $\lim _{x \rightarrow 0} c(x)=c \neq 0, \lim _{x \rightarrow 0} \bar{\varepsilon}(x)=0$ and $a>1$.

The above lemma follows immediately if we put $1 / x$ for $x$ in Karamata's theorem (see Ibragimov and Linnik (1972), Appendix 1, page 394).

Since the random variables belong to the domain of attraction of the normal law, their common characteristic function admits the representation

$$
\phi(t)=\exp \left\{-\frac{1}{2} t^{2} L(|t|)(1+o(1))\right\}
$$

(see Ibragimov and Linnik (1972) Chapter 2 page 91), where, as $t \rightarrow 0, L(t)$ is a slowly varying function. Since $L(|t|)$ is positive we can write the characteristic function $\phi$ in the form

$$
\phi(t)=\exp \left\{-\frac{1}{2} t^{2} h(t)\right\}
$$

where $h(t)=L(|t|)(1+o(1))$ with the property that

$$
h(t)=\operatorname{Re} h(t)(1+o(1)) .
$$

Obviously $h(t)$ is a slowly varying function as $t \rightarrow 0$. 
We know $L(1 / x)$ is given by the formula

$$
L(1 / x)=-\int_{0}^{x} u^{2} d \psi(u)=\int_{-x}^{x} u^{2} d G(u)
$$

where $\psi(x)=1-G(x)+G(-x), G(x)$ being the common distribution function of the random variables $X_{r}$ 's (see Ibragimov and Linnik (1972) Chapter 2, page 90). Hence for infinite variance $\lim _{x \rightarrow 0} L(1 / x)=\infty$ which gives

$$
\lim _{t \rightarrow 0} \operatorname{Re} h(t)=\infty \text {. }
$$

Consider the function $h_{1}(t)$ determined by

$$
h_{1}(t)= \begin{cases}\operatorname{Re} h(t) & \text { if } V\left(X_{r}\right)=\infty, \\ \sigma^{2} & \text { if } V\left(X_{r}\right)=\sigma^{2}<\infty\end{cases}
$$

Clearly $h_{1}(t)$ is slowly varying in a neighbourhood of the origin. By (2.10), $h(t)=h_{1}(t)(1+o(1))$ in both cases as $t \rightarrow 0$.

We define normalising constants $V_{m}$ starting from the relation

$$
\left(1 / V_{m}^{2}\right) \sum_{\mathrm{l}} a_{r}^{2} x_{m}^{2 r} h_{1}(\eta \theta)=1
$$

where $\eta=a_{r} x_{m}^{r} / V_{m}$, and $\theta$ is a small positive number whose final choice will be dealt with later. Proceeding as Ibragimov and Maslova (1971) page 232, we can show that such constants $V_{m}$ always exist if $\theta$ is sufficiently small.

LEMMA 2. For some constant $b>1$ we have

$$
M^{m}< \begin{cases}(A e / D)^{1 / 2}\left(V_{m} / \sigma t_{n}\right) & \text { if } V\left(X_{r}\right)=\sigma^{2}<\infty, \\ b^{-1 / 2}(A e / D)^{1 / 2}\left(V_{m} / t_{n}\right) & \text { if } V\left(X_{r}\right)=\infty\end{cases}
$$

Proof. If $V\left(X_{r}\right)=\sigma^{2}<\infty$, then

$$
V_{m}^{2}=\sigma^{2} \sum_{1} a_{r}^{2} x_{m}^{2} \geqslant \sigma^{2} t_{n}^{2} M^{2 m}(D / A e)
$$

Or

$$
M^{m}<(A e / D)^{1 / 2}\left(V_{m} / \sigma t_{n}\right) .
$$

Again let $V\left(X_{r}\right)=\infty$. Then by (2.11), $\lim _{t \rightarrow 0} h_{1}(t)=\infty$, so we can choose $\theta_{0}>0$ such that for $\eta \theta<\theta_{0}, h_{1}(\eta \theta)>b>1$ where $b$ is a constant and $0 \leqslant r \leqslant n$. Then we have

$$
V_{m}^{2}>b \sum_{1} a_{r}^{2} x_{m}^{2 r} \geqslant b t_{n}^{2} \sum_{1} x_{m}^{2 r}>b t_{n}^{2} M^{2 m}(D / A e)
$$

Or

$$
M^{m}<b^{-1 / 2}(A e / D)^{1 / 2}\left(V_{m} / t_{n}\right)
$$


LEMma 3.

$$
\left|\sum_{2} a_{r} X_{r}(u) x_{m}^{r}\right|<\lambda_{n} W_{m}
$$

except for a set of measure at most $\mu / \lambda_{n}^{2-\varepsilon}$ for $\varepsilon>0$, where

$$
W_{m}^{2}=\sum_{2} a_{r}^{2} x_{m}^{2 r} h_{1}\left(\eta_{1} \theta_{1}\right),
$$

$\eta_{1}=a_{r} x_{m}^{r} / W_{m}$ and $\theta_{1}$ has similar meaning to $\theta$.

PRoof. Let $\psi_{m}(x)$ and $\phi_{m}(t)$ be respectively the distribution function and the characteristic function of $a_{r} X_{r}(u) / W_{m}$.

So $\phi_{m}(t)=\exp \left(-\frac{1}{2} t^{2} h_{m}(t)\right)$, where

$$
h_{m}(t)=\left(1 / W_{m}^{2}\right) \sum_{2} a_{r}^{2} x_{m}^{2 r} h\left(\eta_{1} t\right) .
$$

Since $h_{1}(t)=L(|t|)(1+o(1))$ and $L(|t|)$ is a slowly varying function as $t \rightarrow 0$, we have by Lemma 1 , for $|t|<\theta$,

$$
\begin{aligned}
h_{1}\left(\eta_{1} t\right)\left(h_{1}\left(\eta_{1} \theta_{1}\right)\right)^{-1} & =\frac{L\left(\left|\eta_{1} t\right|\right)(1+o(1))}{L\left(\left|\eta_{1} \theta_{1}\right|\right)(1+o(1))} \\
& =\frac{c\left(\left|\eta_{1} t\right|\right) \exp \left\{-\int_{a}^{\left|\eta_{1} t\right|}(\bar{\varepsilon}(u) / u) d u\right\}(1+o(1))}{c\left(\left|\eta_{1} \theta_{1}\right|\right) \exp \left\{-\int_{a}^{\left|\eta_{1} \theta_{1}\right|}(\bar{\varepsilon}(u) / u) d u\right\}(1+o(1))} \\
& =\frac{c\left(\left|\eta_{1} t\right|\right)(1+o(1))}{c\left(\left|\eta_{1} \theta_{1}\right|\right)(1+o(1))} \exp \left\{\int_{\left|\eta_{1} t\right|}^{\left|\eta_{1} \theta_{1}\right|}(\bar{\varepsilon}(u) / u) d u\right\},
\end{aligned}
$$

where $\lim _{u \rightarrow 0} c(u)=c \neq 0, \lim _{u \rightarrow 0} \bar{\varepsilon}(u)=0$ and $a>0$. Now since $\lim _{u \rightarrow 0} \bar{\varepsilon}(u)$ $=0$, we have for $\varepsilon>0$, there exists a positive $t_{0}$ such that for $|t| \leqslant \theta_{1}<t_{0}^{-1}$,

$$
\exp \left\{\int_{\left|\eta_{1} t\right|}^{\left|\eta_{1} \theta_{1}\right|} \frac{\bar{\varepsilon}(u)}{u} d u\right\}<\exp \left\{\int_{\left|\eta_{1} t\right|}^{\left|\eta_{1} \theta_{1}\right|} \frac{\varepsilon}{u} d u\right\}=\left|t / \theta_{1}\right|^{-\varepsilon}
$$

Since $c(t)=(1+o(1)) c$ as $t \rightarrow 0$, we get

$$
h_{1}\left(\eta_{1} t\right)\left(h_{1}\left(\eta_{1} \theta_{1}\right)\right)^{-1} \leqslant\left|t / \theta_{1}\right|^{-\varepsilon}(1+o(1))
$$

which gives

$$
h_{1}\left(\eta_{1} t\right) \leqslant\left|t / \theta_{1}\right|^{-\varepsilon} h_{1}\left(\eta_{1} \theta_{1}\right)(1+o(1)) .
$$

Hence by virtue of (2.15), (2.16) and (2.17) we have $\operatorname{Re} h_{m}(t) \leqslant\left|t / \theta_{1}\right|^{-\varepsilon}$. Again by (3.10), we have

$$
h_{m}(t)=\operatorname{Re} h_{m}(t)(1+o(1)) \quad \text { as } t \rightarrow 0 .
$$

Therefore, for $|t|<t_{0}^{-1}$ and $\varepsilon>0$, we have $\left|h_{m}(t)\right|<\mu_{1}|t|^{-\varepsilon}$, whence

$$
\left|\phi_{m}(t)-1\right|=\left|\exp \left(\frac{1}{2} t^{2} h_{m}(t)\right)-1\right| \leqslant \mu_{1}|t|^{2-\varepsilon} \text {. }
$$


Now by Gnedenko and Kolmogorov (1968) Chapter 2, page 54, we have

$$
\begin{aligned}
\operatorname{Pr}\left\{\left|\sum_{2} a_{r} X_{r} x_{m}^{r}\right| \geqslant \lambda W_{m}\right\}=1-\left(\psi_{m}\left(\lambda_{n}\right)-\psi_{m}\left(-\lambda_{n}\right)\right) \\
\leqslant 2-\left|\left(\lambda_{n} / 2\right) \int_{-d}^{d} \phi_{m}(t) d t\right| \quad\left(\text { where } d=2 / \lambda_{n}\right) \\
\leqslant\left(\lambda_{n} / 2\right) \int_{-d}^{d}\left|\phi_{m}(t)-1\right| d t \leqslant\left(\lambda_{n} / 2\right) \mu_{1} \int_{-d}^{d}|t|^{2-\varepsilon} d t<\mu / \lambda_{n}^{2-\varepsilon} .
\end{aligned}
$$

Hence the result.

LEMMA 4.

$$
\left|\sum_{3} a_{r} X_{r}(u) x_{m}^{r}\right|<\lambda_{n} Z_{m}
$$

except for a set of measure at most $\mu / \lambda_{n}^{2-\varepsilon}$ for $\varepsilon>0$, where

$$
Z_{m}^{2}=\sum_{3} a_{r}^{2} x_{m}^{2 r} h_{1}\left(\eta_{1} \theta_{2}\right)
$$

$\eta_{2}=a_{r} x_{m}^{r} / Z_{m}$ and $\theta_{2}$ has similar meaning to $\theta$.

The proof of this lemma is exactly similar to that of Lemma 3.

LEMMA 5. For a given $m,\left|R_{m}\right|<V_{m}$ except for a set of measure at most $\mu / \lambda_{n}^{2-\varepsilon}$ for $m=[k / 2]+1,[k / 2]+2, \ldots, k$.

Case I. Let $V\left(X_{r}\right)=\infty$. Now we choose $\theta$ so that for $0 \leqslant r \leqslant n,(\eta \theta)<$ $\min \left(\eta_{1} \theta_{1}, \eta_{2} \theta_{2}\right)$ by which

$$
1<h_{1}\left(\eta_{1} \theta_{1}\right) \leqslant b \text { and } 1<h_{1}\left(\eta_{2} \theta_{2}\right) \leqslant b \text {. }
$$

Now the choice of $\theta$ is final and by this choice it follows from Lemma 3 and Lemma 4 that

$$
\left|R_{m}\right|<\lambda_{n} b^{1 / 2} k_{n}\left(\left(\sum_{2} x_{m}^{2 r}\right)^{1 / 2}+\left(\sum_{3} x_{m}^{2 r}\right)^{1 / 2}\right)
$$

except for a set of measure at most $\mu / \lambda_{n}^{2-\varepsilon}$.

Proceeding as Samal and Mishra (1972a) page 527 and using Lemma 3, we can show that $\left|R_{m}\right|<V_{m}$ except for a set of measure at most $\mu / \lambda_{n}^{2-\varepsilon}$.

Case II. Let $V\left(X_{r}\right)=\sigma^{2}<\infty$. Then

$$
\left|R_{m}\right|<\lambda_{n} k_{n} \sigma\left(\left(\sum_{2} x_{m}^{2 r}\right)^{1 / 2}+\left(\sum_{3} x_{m}^{2 r}\right)^{1 / 2}\right) .
$$


As in Case I, we can show that $\left|R_{m}\right|<V_{m}$ except for a set of measure at most $\mu / \lambda_{n}^{2-\varepsilon}$.

\section{Proof of the theorem}

By (2.5) we have

$$
U_{2 m}=\sum_{M^{4 m-1+1}}^{M^{4 m+1}} a_{r} X_{r} x_{m}^{r} \text { and } U_{2 m+1}=\sum_{M^{r m+1}+1}^{M^{4 m+3}} a_{r} X_{r} x_{m}^{r} .
$$

So $X_{r}$ 's in $U_{2 m}$ do not occur in $U_{2 m+1}$. Therefore $U_{2 m}$ and $U_{2 m+1}$ are independent random variables.

We define sets $E_{m}$ and $F_{m}$ as follows:

$$
\begin{aligned}
& E_{m}=\left\{U_{2 m} \geqslant V_{2 m}, U_{2 m+1}<-V_{2 m+1}\right\}, \\
& F_{m}=\left\{U_{2 m}<-V_{2 m}, U_{2 m+1} \geqslant V_{2 m+1}\right\} .
\end{aligned}
$$

Let $G_{m}(x)$ and $g_{m}(t)$ be respectively the distribution function and the characteristic function of $\left(U_{m} / V_{m}\right)$. Then

$$
g_{m}(t)=\exp \left\{-\frac{1}{2} t^{2}\left(1 / V_{m}^{2}\right) \sum_{1} a_{r}^{2} x_{m}^{2 r} h(\eta t)\right\} .
$$

Let

$$
F(x)=(1 / \sqrt{2 \pi}) \int_{-\infty}^{x} \exp \left(-u^{2} / 2\right) d u .
$$

From Lemma 2 it follows that $V_{m} \rightarrow \infty$ as $m \rightarrow \infty$ and so $\eta t \rightarrow 0$ as $m \rightarrow \infty$. Then $h(\eta t)=h_{1}(\eta t)(1+o(1))$. By Lemma 1 , we have in a neighbourhood of the origin,

$$
h_{1}\left(\eta_{t}\right)=h_{1}(\eta \theta)|\theta / t|^{o(1)}(1+o(1)) \text {. }
$$

So when $m \rightarrow \infty$,

$$
\begin{aligned}
g_{m}(t) & =\exp \left\{-\frac{1}{2} t^{2}\left(1 / V_{m}^{2}\right) \sum_{1} a_{r} x_{m}^{2 r} h_{1}(\eta \theta)|\theta / t|^{o(1)}(1+o(1))(1+o(1))\right\} \\
& \left.=\exp \left\{-\frac{1}{2} t^{2-o(1)} \theta^{o(1)}(1+o(1))\right\} \quad \text { (by definition of } V_{m}\right) .
\end{aligned}
$$

Therefore as $m \rightarrow \infty, g_{m}(t) \rightarrow \exp \left(-\frac{1}{2} t^{2}\right)$ in any bounded interval of $t$-values. Hence

$$
\sup _{x}\left|G_{m}(x)-F(x)\right|=o(1) .
$$

So for $\varepsilon>0$, we have

$$
\left|G_{2 m}(-1)-F(-1)\right|<\varepsilon,
$$


and

$$
\left|G_{2 m+1}(-1)-F(-1)\right|<\varepsilon .
$$

Now since $U_{2 m}$ and $U_{2 m+1}$ are independent, we have

$$
\begin{aligned}
\operatorname{Pr}\left(E_{m} \cup F_{m}\right)= & \operatorname{Pr}\left(U_{2 m} \geqslant V_{2 m}\right) \operatorname{Pr}\left(U_{2 m+1}<-V_{2 m+1}\right) \\
& +\operatorname{Pr}\left(U_{2 m}<-V_{2 m}\right) \operatorname{Pr}\left(U_{2 m+1} \geqslant V_{2 m+1}\right) \\
= & G_{2 m+1}(-1)\left(1-G_{2 m}(1)\right)+G_{2 m}(-1)\left(1-G_{2 m+1}(1)\right) .
\end{aligned}
$$

By (3.4) and (3.5), we have $G_{2 m}(-1)>F(-1)-\varepsilon, G_{2 m+1}(-1)>F(-1)-\varepsilon$, and $1-G_{2 m}(1)>1-F(1)-\varepsilon, 1-G_{2 m+1}(1)>1-F(1)-\varepsilon$. Hence $\operatorname{Pr}\left(E_{m} \cup F_{m}\right)$ $\geqslant 2(F(-1)-\varepsilon)(1-F(1)-\varepsilon)$. Thus $\operatorname{Pr}\left(E_{m} \cup F_{m}\right)$ is greater than a quantity which tends to $2 F(-1)(1-F(1))$ as $m \rightarrow \infty$ with $n$. This limit being positive we conclude that for large $m, \operatorname{Pr}\left(E_{m} \cup F_{m}\right)>\delta>0$ where $\delta$ is an absolute constant. Hence $\operatorname{Pr}\left(E_{m} \cup F_{m}\right)=\delta_{m}>\delta>0$.

Let us define random variables $y_{m}$ such that it takes value 1 on $E_{m} \cup F_{m}$ and 0 elsewhere. Define

$$
z_{m}= \begin{cases}0 & \text { if }\left|R_{2 m}\right|<V_{2 m} \text { and }\left|R_{2 m+1}\right|<V_{2 m+1} \\ 1 & \text { otherwise. }\end{cases}
$$

Proceeding as in Samal and Mishra (1972b) page 560, we can show that the number of roots in the interval $\left(x_{2 m_{0}}, x_{2 k+1}\right)$ must exceed $\sum_{m=m_{0}}^{k}\left(y_{m}-y_{m} z_{m}\right)$, where $m_{0}=[k / 2]+1$ and also

$$
\begin{aligned}
E\left(\sum y_{m} z_{m}\right) & \leqslant \sum \operatorname{Pr}\left\{\left(\left|R_{2 m}\right| \geqslant V_{2 m}\right) \cup\left(\left|R_{2 m+1}\right| \geqslant V_{2 m+1}\right)\right\} \\
& <(k+1)\left(\mu / \lambda_{n}^{2-\varepsilon}\right) \quad \text { (by Lemma 5). }
\end{aligned}
$$

Hence for $0<\beta<2-\varepsilon$,

$$
\operatorname{Pr}\left\{\sum y_{m} z_{m}>(k+1) \lambda_{n}^{\beta}\left(\mu / \lambda_{n}^{2-\epsilon}\right)\right\}<\frac{E\left(\sum y_{m} z_{m}\right)}{(k+1) \lambda_{n}^{\beta}\left(\mu / \lambda_{n}^{2-\varepsilon}\right)}<1 / \lambda_{n}^{\beta} .
$$

Therefore,

$$
\sum_{m=m_{0}}^{k} y_{m} z_{m}<(k+1)\left(\mu / \lambda_{n}^{2-\varepsilon}\right) \lambda_{n}^{\beta}<\mu^{\prime} k / \lambda_{n}^{2-\varepsilon-\beta} .
$$

As in Samal and Mishra (1972a) page 525, we can show here that $\sum_{m=m_{0}}^{k} y_{m}>\mu k$ outside a set of measure at most $\mu^{\prime} / k$. Therefore

$$
N_{n}>\sum_{m_{0}}^{k}\left(y_{m}-y_{m} z_{m}\right)>\mu k-\left(\mu^{\prime} k / \lambda_{n}^{2-\mathbf{e}^{-\beta}}\right)>\mu_{0} k
$$

outside an exceptional set of measure at most $\mu / k+1 / \lambda_{n}^{\beta}$. From (2.3) we have

$$
\mu_{1}\left(\lambda_{n}\left(k_{n} / t_{n}\right)\right)^{2} \leqslant M \leqslant \mu_{2}\left(\lambda_{n}\left(k_{n} / t_{n}\right)\right)^{2},
$$


and from (2.4), we have

$$
\mu_{3} \frac{\log n}{\log M}<k<\mu_{4} \frac{\log n}{\log M} .
$$

Now from (3.7) and (3.8) we have

$$
\frac{\mu_{5} \log n}{\log \left(\lambda_{n}\left(k_{n} / t_{n}\right)\right)}<k<\mu_{6} \frac{\log n}{\log \left(\lambda_{n}\left(k_{n} / t_{n}\right)\right)} .
$$

Set

$$
\lambda_{n}=\left(t_{n} / k_{n}\right) \exp \left(1 / \varepsilon_{n}^{\prime}\right)
$$

where $\varepsilon_{n}^{\prime}$ has the same meaning as $\varepsilon_{n}$. Obviously $\lambda_{n} \rightarrow \infty$ as $n \rightarrow \infty$. From (3.10) it follows that

$$
\mu_{5} \varepsilon_{n}^{\prime} \log n<k<\mu_{6} \varepsilon_{n}^{\prime} \log n
$$

Therefore $k \rightarrow \infty$ as $n \rightarrow \infty$. Again by (3.11), we have

$$
N_{n}>\mu_{7} \varepsilon_{n}^{\prime} \log n=\varepsilon_{n} \log n, \quad \text { where } \mu_{7} \varepsilon_{n}^{\prime}=\varepsilon_{n} .
$$

If $G$ is the exceptional set then

$$
\begin{aligned}
\operatorname{Pr}(G) & <\mu^{\prime} / k+1 / \lambda_{n}^{\beta}<\mu^{\prime} /\left(\mu_{5} \varepsilon_{n}^{\prime} \log n\right)+\left(k_{n} / t_{n}\right)^{\beta} \exp \left(-\frac{\beta}{\varepsilon_{n}^{\prime}}\right) \\
& =\mu^{\prime} /\left(\left(\mu_{5} / \mu_{7}\right) \varepsilon_{n} \log n\right)+\left(k_{n} / t_{n}\right)^{\beta} \exp \left(-\left(\mu_{7} \beta \lambda \varepsilon_{n}\right)\right) \\
& <\mu / \varepsilon_{n} \log n+\left(k_{n} / t_{n}\right)^{\beta} \exp \left(-\left(\mu^{\prime} \beta / \varepsilon_{n}\right)\right) .
\end{aligned}
$$

The authors wish to thank the referee for his valuable comments.

\section{References}

B. V. Gnedenko and A. N. Kolmogorov (1968), Limit distribution of sums of independent random variables, (Addison Wesley, Inc).

G. Samal (1962), 'On the number of real roots of a random algebraic equation', Proc. Cambridge Philos. Soc. 58, 433-442.

G. Samal and M. N. Mishra (1972a), 'On the lower bound of the number of real roots of a random algebraic equation with infinite variance', Proc. Amer. Math. Soc. 33, 523-528.

G. Samal and M. N. Mishra (1972b), 'On the lower bound of the number of real roots of a random algebraic equation with infinite variance II, Proc. Amer. Math. Soc. 36, 557-563.

G. Samal and M. N. Mishra (1973), 'On the lower bound of the number of the real roots of a random algebraic equation with infinite variance III, Proc. Amer. Math. Soc. 39, 184-189.

I. A. Ibragimov and Yu. V. Linnik (1972), Independent and stationary sequences of random variables (Wolters-Noordhoff, Groningen). 
I. A. Ibragimov and N. B. Maslova (1971), 'On the expected number of real zeros of random algebraic polynomials I, Coefficient with zero means' (translated by B. Seckler), Theory of Probability and its Applications, Vol. XVI, 2, 228-248.

J. E. Littlewood and A. C. Offord (1939), ' On the number of real roots of a random algebraic equation II', Proc. Cambridge Philos. Soc. 35, 133-148.

J. E. A. Dunnage (1978), 'The number of real roots of a class of random algebraic polynomial', Proc. London Math. Soc. (3) 18, 439-460.

J. E. A. Dunnage (1970), 'The number of real zeros of a class of random algebraic polynomial II', Quart. J. Math. Oxford Ser. (2) 21, 309-319.

J. E. A. Dunnage (1972), 'The number of real zeros of a class of random algebraic polynomial III', $J$. London Math. Soc. (2) 6, 143-152.

School of Mathematical Sciences

Sambalpur University

Jyoti Vihar, Burla, 768017

Orissa India
College of Basic Science and Humanities, O.U.A.T. Bhubaneswar 751005 Puri, Orissa India 